\title{
Transpiration actuation: the design, fabrication and characterization of biomimetic microactuators driven by the surface tension of water
}

\author{
Ruba T Borno, Joseph D Steinmeyer and Michel M Maharbiz \\ Department of Electrical Engineering and Computer Science, University of Michigan, \\ Ann Arbor, MI 48109, USA
}

Received 14 June 2006, in final form 15 August 2006

Published 21 September 2006

Online at stacks.iop.org/JMM/16/2375

\begin{abstract}
We have designed, fabricated and characterized large displacement distributed-force polymer actuators driven only by the surface tension of water. The devices were inspired by the hygroscopic spore dispersal mechanism in fern sporangia. Microdevices were fabricated through a single mask process using a commercial photo-patternable silicone polymer to mimic the mechanical characteristics of plant cellulose. An analytical model for predicting the microactuator behavior was developed using the principle of virtual work, and a variety of designs were simulated and compared to the empirical data. Fabricated devices experienced tip deflections of more than $3.5 \mathrm{~mm}$ and angular rotations of more than $330^{\circ}$ due to the surface tension of water. The devices generated forces per unit length of $5.75 \mathrm{mN} \mathrm{m}^{-1}$ to $67.75 \mathrm{mN} \mathrm{m}^{-1}$. We show initial results indicating that the transient water-driven deflections can be manipulated to generate devices that self-assemble into stable configurations. Our model shows that devices should scale well into the submicron regime. Lastly, the actuation mechanism presented may provide a robust method for embedding geometry-programmable and environment-scavenged force generation into common materials.
\end{abstract}

(Some figures in this article are in colour only in the electronic version)

\section{Introduction}

There is an increasing desire to embed actuation into normally inert materials for smart structural components and selfassembling manufacturing applications [1]. Additionally, technology miniaturization into the micro and nanoscale and the increasing decentralization of sensing and computation have created a need for novel energy-scavenging technologies [2]. Devices that scavenge energy or perform work by utilizing energy already present in their environment are of interest to MEMS researchers [3]. Potential sources of ambient energy include, but are not limited to, vibrations [4] and wind [5]. The work presented here focuses on the use of environmental light or heat for evaporation-induced actuation driven by surface tension.
In addition to energy scavenging, researchers have demonstrated an interest in the potential for extracting work from liquid surface tension for use in bottom-up selfassembly [6-8], batch assembly of microcomponents [9-12] and actuation [13, 14]. Previously, the surface tension of liquid solder has been used to assemble large devices for electrical networks and three-dimensional elastomer structures [15, 16]. Metal solder pads were first patterned and then melted to rotate adjacent structures using the surface tension of the molten solder. Surface tension has also been explored as a possible method to drive low-friction actuation [13]. A bistable liquid-solid micromechanical contact switch was made from a 5-20 $\mu \mathrm{m}$ diameter droplet that maintained its structural stability due to the surface tension of the liquid. In addition to the use of liquid metals for actuation, the surface 
tension of water has been exploited to study the mechanical strength of lithographic polymers [17]. Photo-defined test structures consisting of narrow, parallel beams were developed and released in water. As the channels between the test structures dried, water tension caused stiction in some devices. The Young-Laplace equation governed the initial pressure difference between the test structures and was used to calculate the polymer strength.

Interestingly, the ability to extract work from both environmental conditions and liquid surface tension is a hallmark of plant evolution [18-21]. Here, we present a class of biomimetic microactuators inspired by hygroscopic spore dispersal mechanisms in ferns. We previously introduced the first generation of these biomimetic actuators in [22]. The following text elaborates on the microactuators which scavenge energy from evaporating water to generate geometrically programmable force profiles and deflections. This text also describes an analytical model to predict actuator performance. The presented transpiration actuation mechanism enables the infusion of common materials with distributed programmable force generation and may also form the basis of a means to scavenge energy from environmental humidity.

This paper begins, in section 2, with a description of the device geometry and parameters. Section 3 details the analytical model of device operation based on energy methods. Section 4 describes the method by which the biomimetic actuators were fabricated and tested. Section 5 presents the empirical test results of the fabricated devices and compares them to the theoretical model; the significance of various geometric design parameters on the amount of actuation, angle of rotation and the effect of scaling are discussed. Lastly, we show how this transient evaporation actuation can be used to self-assemble devices into stable geometries.

\subsection{Fern sporangia}

Plants have evolved methods for extracting work from the surface tension of water. One example is the pumping of water from root to leaves via evaporation at microscale stomatal pores, known as the cohesion-tension theory [1821]. Similarly, ferns make use of water evaporation within specialized microstructures to obtain fast motion and high forces for spore dispersal [21]. Ferns grow specialized reproductive vessels called sporangia (figure $1(a)$ ). During the dry season, these microscale sporangia open violently to release small spores into the air (figure 1(b)). Each sporangium is surrounded by an annulus of water-filled cells and each cell comprises two rib-like structures filled with water [23]. As water dries inside the cells, the surface tension between the water and the cell wall gives rise to high forces causing a deflection along the outer edge of the annulus. The combined deflection of each wall straightens the entire annulus structure and tears open the spore sac (figure $1(b)$ ).

\subsection{Surface tension at the microscale}

Due to the high surface area-to-volume ratio, surface tension is a dominant force at the micro and nanoscale [14, 24, 25]. In the bulk, the potential energy of a water molecule, where its forces are counterbalanced by those of its surrounding molecules, is

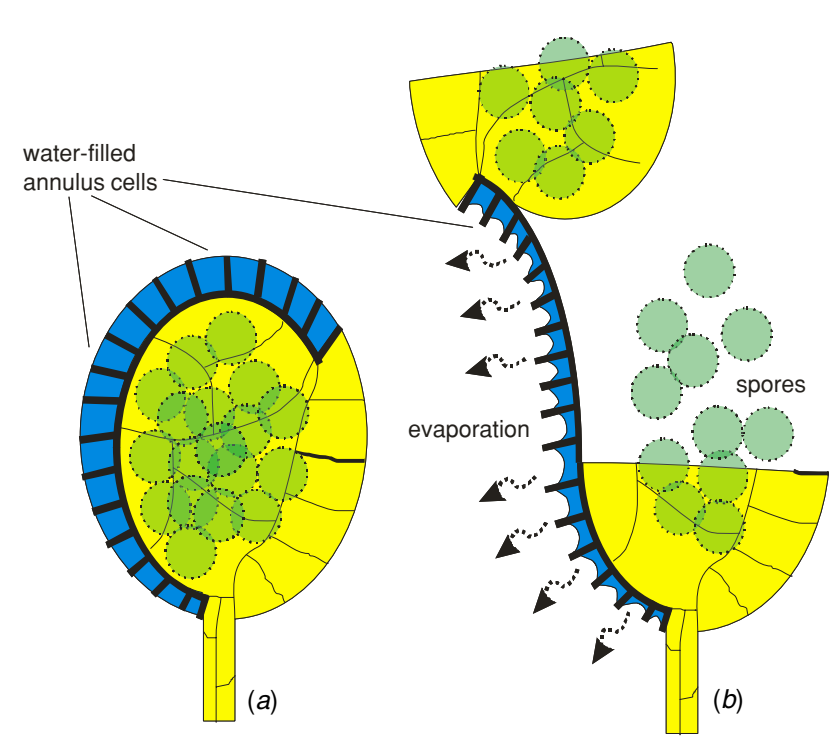

Figure 1. Actuation mechanism of fern sporangium: (a) before and (b) after evaporation-induced opening of annulus to release spores (adapted from [21]).

minimized [24]. At the liquid-air interface on the surface, the forces of each water molecule are left unbalanced. Because of the high energy at the liquid-air interface, liquids seek to minimize surface area. This mechanism is the premise upon which the devices presented in this paper operate; water contained between two walls seeks to minimize its liquidair surface area as it evaporates, thus causing mechanical deformation.

\section{Design}

The devices designed in this work mimic the geometries of fern sporangia (figure 2(a)). Devices consisted of a curved spine that straightened during the device operation. The spine was also designed around the physics of a slender beam. Ribs extending from the spine create cells with two sidewalls which can be filled with water. These ribs act as levers upon which forces due to surface tension act to deform the spine (figures $2(b)$ and $(c)$ ).

Both the spine and ribs were made from silicone polymer. In order to mimic the mechanical properties of plant cellulose, polydimethylsiloxane (PDMS) was used because of its comparable Young's modulus. Cellulose, the material that comprises plant cell walls, has a Young's modulus of 120 $500 \mathrm{MPa}$ [26]. Young's modulus of the photo-patternable silicone employed in this work was $160 \mathrm{MPa}$, as reported by the manufacturer.

The behavior of these devices can be compared to previous solder-driven self-assembling plates. There has been much previous work on surface tension actuation utilizing solder at high temperatures $[7,8,15,16]$. As the solder melts, the liquid minimizes its energy by reducing the interfacial surface area (figure $2(d)$ ). The surface tension forces at the solder-plate interface cause the hinged plate to rotate as the liquid-solder surface energy is minimized. The microactuators in the work presented in this paper employ a distributed energy minimization mechanism by including multiple liquid 


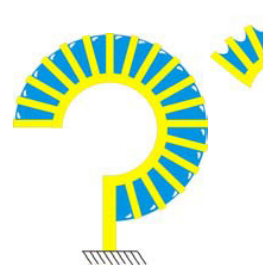

(a)

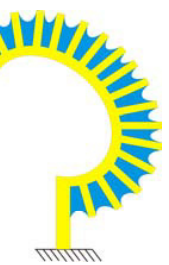

(b)

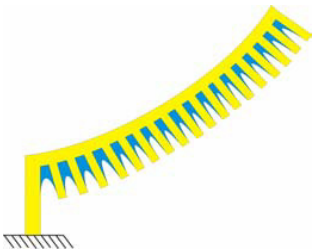

(c)

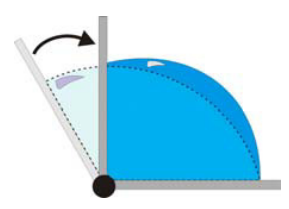

(d)

Figure 2. Actuation mechanism of microfabricated devices: (a) water is placed between the ribs to wet the device. (b) Immediately after wetting, the surface tension of the water pulls on the ribs at the meniscus edge. $(c)$ As the water seeks to minimize its surface energy, surface tension pulls on the ribs and causes the spine to deform. $(d)$ Energy minimization has been used to rotate hinges using molten solder as presented in $[8$, figure $3(d)]$. The device in $(a)-(c)$ multiplies the energy minimization effect by incorporating the ribs to divide the liquid volume.

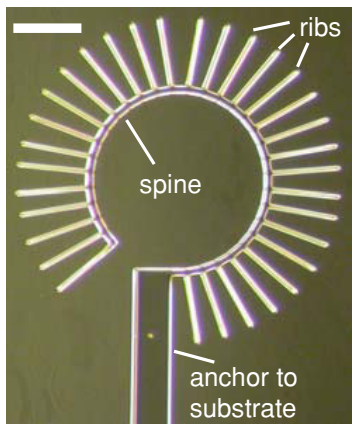

(a)

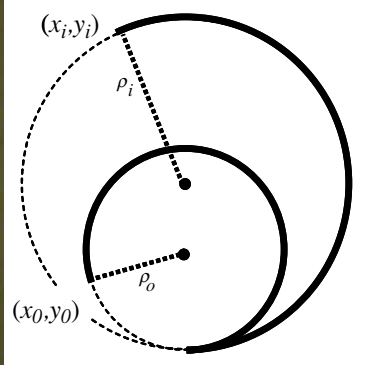

(b)

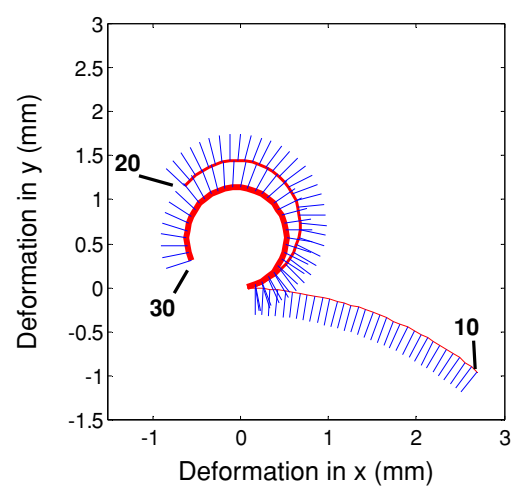

(c)

Figure 3. (a) A micrograph of a device in its initial rest state. The scale bar indicates $400 \mu \mathrm{m}$. (b) An illustration of how the strain energy of the spine is calculated, using a constant arc length with an increasing radius of curvature $\rho$. Tip deflections, $\delta$, are calculated as $\delta=\sqrt{\left(x_{i}-x_{0}\right)^{2}+\left(y_{i}-y_{0}\right)^{2}}$. (c) Simulated deformation curves at the point of equilibrium between the strain energy of the spine and the surface energy of the water for devices with varying spine thicknesses (10,20 and $30 \mu \mathrm{m}$ ) and a rib length of $400 \mu \mathrm{m}$. As the spine thickness is decreased equilibrium is reached at higher deflections.

volumes that seek to reduce their surface energies. As will be explained, this distribution of actuation is useful for achieving additional control and for embedding force-generation profiles into the actuation.

\section{Analytical model}

\subsection{Principle of virtual work}

In this section, we use the principle of virtual work as the basis of an analytical model that predicts device performance. We first describe the principle of virtual work and describe the energy components. We then calculate the strain energy due to deformation of the spine and the surface energy of the liquid between the device ribs. Those values are then used to find the minimum total potential energy.

The task of predicting the exact nature of device deformations using force balance methods can be quite challenging for even the simple geometries presented above. It is increasingly possible to use finite element models and commercial solvers to approach problems including surface tension effects, large deformation analysis and nonlinear material properties. However, these simulations are typically computationally expensive since there are no obvious symmetries to exploit at the global level and the models do not always converge. In this light, energy methods based on the principle of virtual work are particularly useful. Such methods have been used extensively to model MEMS devices [27]. The model presented here provides an approximate solution to the maximum achievable device deformation based on geometric parameters.

In the present context, the principle of virtual work states that when a body in equilibrium experiences external forces that cause deformation, the energy added to the deformed body is solely due to those applied forces [27, 28]. In this analysis, all forces other than surface tension are neglected. The total potential energy of the microactuators presented here is the sum of the deformation energy of the spine and the surface energy of the water column between the ribs. The model measures the total energy of the various resting states of the device when the ribs are completely filled with water and seeks to find the state of minimum potential energy. In the ideal case, there are no fabrication defects, the ribs are distributed evenly along the spine, each of the cells is completely filled with water, and the evaporation rate for each cell is the same at any given time. The model is further based on the following statements.

(1) The model seeks to find the equilibrium state of the system. Transient response is not being modeled.

(2) Devices are designed to have an initial stress-free shape that is curved. The energy of the stress-free curve is zero.

(3) The model assumes no shear forces and no out-of-plane distortion.

(4) We designed the spine to have uniform radius of curvature in its stress-free shape. The assumption of no shear 


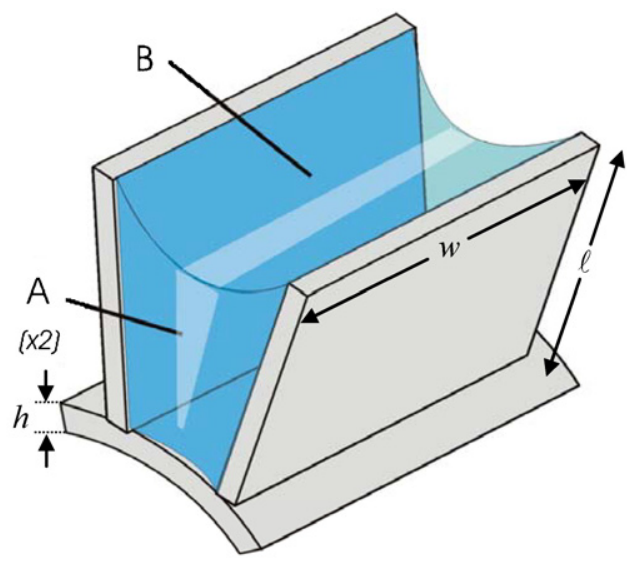

(a)

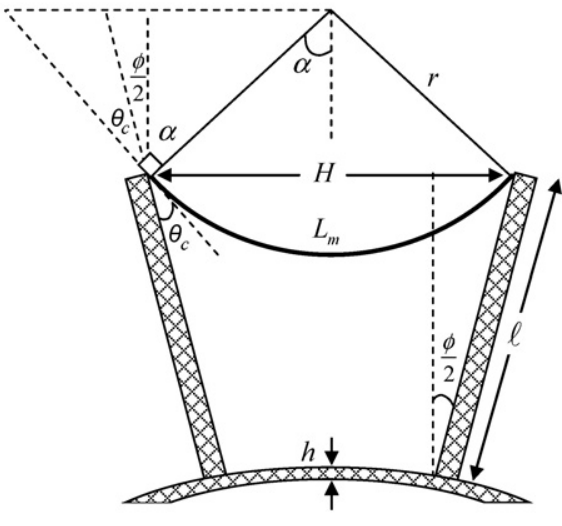

(b)

Figure 4. (a) The two components in the total surface energy of water: area $A$ indicates the side area of the meniscus which depends on the rib length $\ell(\times 2$ to account for the front and back areas) and area $B$ indicates the area of the meniscus above the ribs which depends on the device depth $w$. (b) Variables used in the calculation of the length of the meniscus $L_{m}: r$ is the radius of curvature of the meniscus between two ribs, $H$ is the distance between the tips of the ribs, $\theta_{c}$ is the contact angle between water and the rib, $2 \alpha$ is the angle sweeping the arc length of the meniscus and $\phi$ is the angle between two ribs.

implies that any deformed shape of the spine will also have uniform radius of curvature (figure $3(b)$ ).

(5) The ribs are assumed to be rigid so that they do not store any deformation energy.

As the device deforms due to surface tension the strain energy in the spine increases. Equilibrium is reached when the total potential energy is at a minimum.

\subsection{Strain energy of the spine}

The strain energy is defined as the integral of a scalar effort, modeled by stress, over a scalar displacement, modeled by strain [15]. The spine of the microactuator presented here can be modeled as a continuous curved beam. The strain energy density of the spine is modeled by $\tilde{W}$ in (1), where $E$ is Young's modulus and $\varepsilon$ is the strain:

$$
\tilde{W}(x, y, z)=\int_{0}^{\varepsilon(x, y, z)} E \varepsilon \mathrm{d} \varepsilon=\frac{1}{2} E[\varepsilon(x, y, z)]^{2} .
$$

For large deformations in polymers, nonlinear expressions from neo-Hookean and Mooney-Rivlin analyses can be used to include additional nonlinear terms to the axial stress-strain relationship [29, 30]. However, the maximum strains of the geometries presented in this work do not exceed $\sim 0.1$ for spine thicknesses $<100 \mu \mathrm{m}$. Due to the small strains of the device geometries, a linear axial stress-strain relationship can be used (Hooke's law) [29, 31].

In order to get the energy stored in the beam, the expression must be integrated over the volume of the segment of the spine that is being analyzed. The strain energy of a spine with the rest shape of a curve with radius $\rho_{0}$ is given by equation (2):

$$
W_{\text {strain }}=\frac{E h^{3} w L}{24}\left(\frac{1}{\rho}-\frac{1}{\rho_{0}}\right)^{2},
$$

where $E$ is Young's modulus of the spine material and $h$ and $w$ define the rectangular cross-section of the spine in figure 4 , such that $w$ is the depth of the device into the page and $h$ is the in-planar thickness of the spine. $L$ is the length of the spine, $\rho_{0}$ is the initial radius of curvature of the stress-free spine and $\rho$ is the radius of curvature of the deformed spine [32]. The stress-free curvature is given by (3) and the bending stiffness is given by (4),

$$
\begin{gathered}
\frac{1}{\rho_{0}}=\frac{\theta_{\text {initial }}}{L} \\
E I=\frac{E h^{3} w}{12},
\end{gathered}
$$

where $\theta_{\text {initial }}$ is the angle of the arc swept by the curved spine and $I$ is the moment of inertia of the spine.

The strain energy is based on the physics of a straight cantilever. This is the opposite of the actuator geometry which is curved (figure $3(a)$ ). Although there are cases in which an initially curved beam cannot be modeled using the altered equations for a straight beam, it is acceptable in this case. For the devices presented here, the calculated neutral axis radius of the straight spine is within $0.05 \%$ of the geometrically calculated curved spine value using spine thicknesses, $h<$ $50 \mu \mathrm{m}$. Therefore, the energy present in an initially curved beam that straightens during actuation is essentially equivalent to the strain energy in an initially straight beam that is curved during actuation.

\subsection{Surface energy of water}

As previously mentioned, the system is in the equilibrium state when its total energy, comprising strain energy and surface energy, is at a minimum. The actual surface of the volume of water filling in the region between adjacent ribs is a complex $3 \mathrm{D}$ shape. This surface has been approximated as a set of trapezoids for the pair of 'side' areas which touch both the adjacent ribs and the spine (figure $4(a)$, area $A$ ) and as a partial cylinder of appropriate radius for the 'top' areas which touch just the adjacent ribs (figure $4(a)$, area $B$ ). The sum of these two regions gives an approximation of the surface area of the volume of the water present between the ribs. The work done 


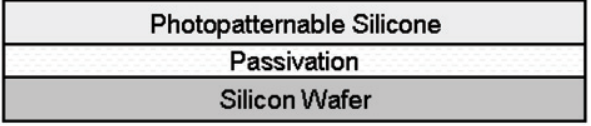

(a)

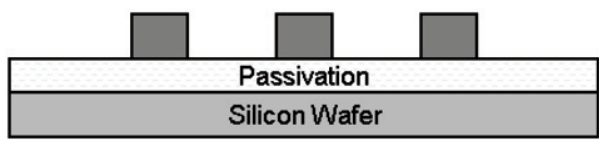

$(c)$

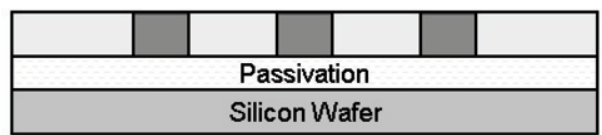

(b)

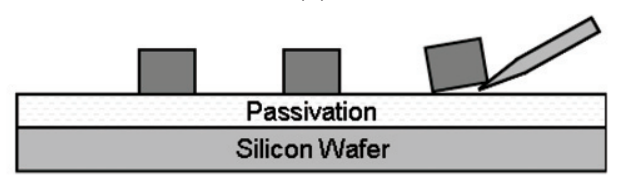

(d)

Figure 5. Fabrication sequence of lithographically patterned polymer actuators: $(a)$ a thin passivation layer is deposited on a bare wafer and photo-patternable silicone is spun on top. $(b)$ Negative photolithography is used to activate polymerization of the silicone. $(c)$ The silicone is developed. $(d)$ The patterned devices are removed from the wafer.

by the surface tension of water for a given spine deformation is expressed as (5)

$$
W_{\text {surface }}=2\left(\sum_{0}^{n} \gamma_{t} \cdot A\right)+\sum_{0}^{n} \gamma_{t} \cdot B,
$$

where $\gamma_{t}$ is the surface tension of water, $A$ is the area of the side meniscus, $B$ is the area of the top meniscus between the tips of a set of ribs and $n$ is the number of ribs. The area of the side meniscus, $A$, can be approximated by a trapezoid that depends on the length of the rib, $\ell$ (6). The curvature of the surface due to the meniscus of the water is neglected for cases where the depth of the rib, $w$, is much greater than the distance between the tips of the ribs, $H$. The partial cylinder used to approximate the 'top' area, area $B$, takes into account the menisci generated by the liquid-solid interface. The length of the meniscus, $L_{m}$, within each cell from rib tip to rib tip can be solved using the water-to-rib contact angle, $\theta_{c}$, and a geometric construction (7)-(9) (figure 4(b)), where $r$ is the radius of curvature of the meniscus between two ribs, $\phi$ is the angle between two ribs, $H$ is the distance between the tips of the ribs and $w$ is the depth of the ribs (figure $4(a)$ ). The approximation for the 'top' surface area, $B$, subtracts the area that does not contain water due to the shape of the meniscus along the depth of the ribs, $w$, in figure $4(a)$. $B$ also subtracts the area due to the meniscus at the very tip of the ribs coming from the side of the volume of the water (10):

$$
\begin{gathered}
A=\ell\left(H-\tan \frac{\phi}{2}\right) \\
\alpha=\frac{\pi}{2}-\frac{\phi}{2}-\theta_{c} \\
r=\frac{H / 2}{\sin (\alpha)} \\
L_{m}=2 \alpha \cdot r \\
B=L_{m} \cdot w-2\left(\alpha r^{2}-r^{2} \sin \alpha \cos \alpha\right) .
\end{gathered}
$$

In equation (10) the unadjusted partial cylinder area is given in the first term and the subtracted areas due to the menisci are given in the second term. These approximations result in a value for the total surface area of the water contained within a set of ribs which takes into account the contact angle of water to the ribs.

\section{Experimental methods}

\subsection{Microfabrication}

The microdevices were batch fabricated out of the photopatternable silicone WL-5150 from Dow Corning using a single mask process. Figure 5 shows the process steps in the fabrication sequence. A passivation layer was deposited on a clean wafer using a Surface Technology Systems ICP-DRIE tool for $1 \mathrm{~min}$ with $85 \mathrm{sccm}$ of $\mathrm{C}_{4} \mathrm{~F}_{8}$ at $800 \mathrm{~W}$ power. This layer facilitates the removal of the silicone structures once fabricated. The silicone was warmed to room temperature and was spun onto the passivated wafer at $300 \mathrm{rpm}$ for a depth of $80 \mu \mathrm{m}$. The coated wafer was then soft baked on a hotplate at $110{ }^{\circ} \mathrm{C}$ for $2 \mathrm{~min}$ to remove carrier solvents. The patternable silicone layers were exposed to $1000 \mathrm{~mJ} \mathrm{~cm}^{-2}$ of UV light using a GCA Autostep 200 i-line stepper to activate polymerization. The exposed silicone layers were then baked on a hotplate for $2 \mathrm{~min}$ at $150^{\circ} \mathrm{C}$ to achieve full polymerization of the exposed regions. Stoddard solvent was used to remove the unexposed silicone during a 1 min puddle development. Fresh developer was deposited onto the wafers with a pipette for $10 \mathrm{~s}$ as they were spun at $200 \mathrm{rpm}$. Isopropyl alcohol was then squirted onto the wafers while spinning them at $500 \mathrm{rpm}$ to remove solvents and undeveloped silicone. Subsequently, wafers were spun dry for $30 \mathrm{~s}$ at $500 \mathrm{rpm}$. Final silicone features were cured on a hotplate at $250{ }^{\circ} \mathrm{C}$ for $10 \mathrm{~min}$. The devices were separated from their carrier wafers using a razor blade. Next, they were immersed in methanol and dried on a clean silicon wafer. Following this, the devices were lifted from the wafer and mounted at their base anchor for testing. Finally, a $50 \mathrm{~W}, 2$ min $\mathrm{O}_{2}$ plasma treatment at 250 mTorr was used to make the structures hydrophilic prior to testing.

\subsection{Testing}

Following the $\mathrm{O}_{2}$ plasma step, water was applied onto the hydrophilic surfaces using a needle and a syringe. Evaporation took place at room temperature. Device actuation was observed and recorded using a Nikon Coolpix 5 Megapixel digital camera mounted on a compound microscope using $10 \times$ magnification. In some cases, $10 \mu \mathrm{M}$ fluorescein (Sigma-Aldrich Corporation) was dissolved in DI water to help visualize the water and the meniscus. 


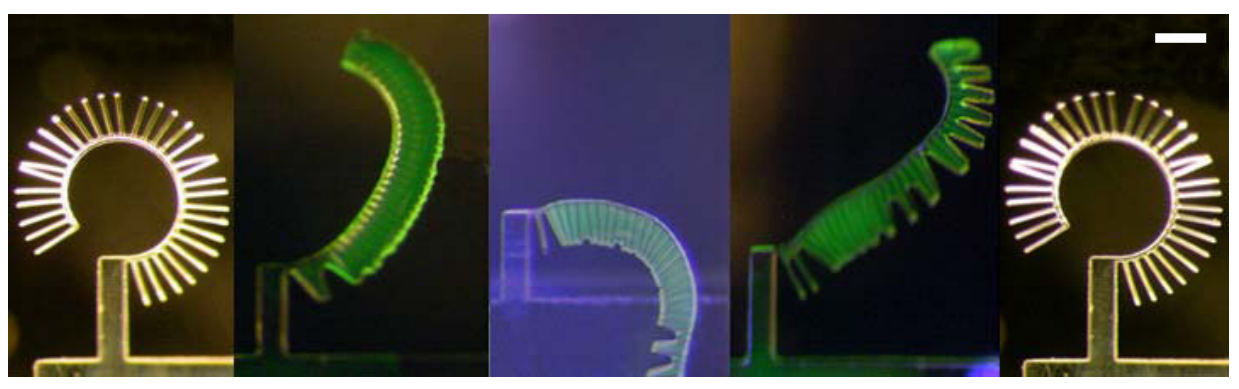

(a)

(b)

(c)

(d)

(e)

Figure 6. Photos of device unfurling: (a) completely dry device before filling. $(b)$ Wetted device $0.5 \mathrm{~s}$ after filling. (c) Device at full deflection $2 \mathrm{~s}$ after filling. (d) Device drying at $30 \mathrm{~s}$ after filling. (e) The device returns to its original profile once water evaporates completely. Note that $(e)$ appears to be identical to $(a)$, but they are in fact completely different images taken before and after actuation. $10 \mu \mathrm{M}$ of fluorescein was added to the water to help visualize the meniscus. The scale bar indicates $400 \mu \mathrm{m}$.

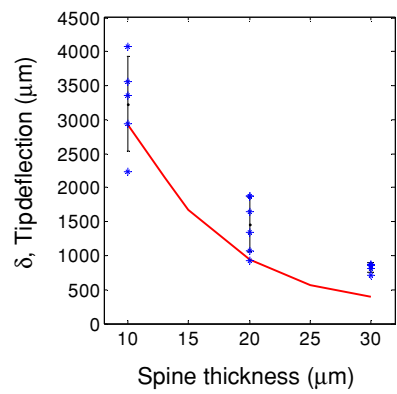

(a)

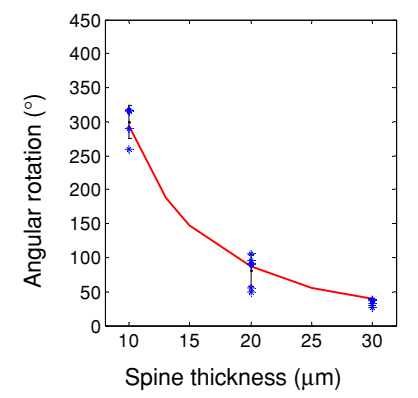

(b)

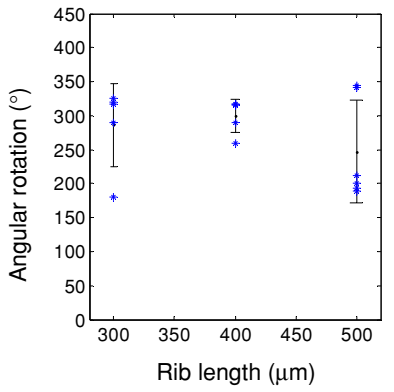

(c)

Figure 7. (a) Tip deflection, $\delta$, defined in figure 3 at the tip of the device as a function of the spine thickness (rib length $=400 \mu \mathrm{m}$ ). (b) Angle of rotation as a function of the spine thickness (rib length $=400 \mu \mathrm{m}$ ). (c) Angle of rotation as a function of the rib length (spine thickness $=10 \mu \mathrm{m}$ ). Vertical lines indicate one standard deviation of the measured results. An angular rotation of $330^{\circ}$ indicates a device actuated from the undeformed arc into a straight line. The results of the simulation are given in red for $60 \mathrm{MPa}$.

\section{Results}

\subsection{Device performance metrics}

Device performance was quantified in two ways: device tip deflection, measured as the distance that the tip moved from its initial position to the point of equilibrium, and angular rotation, which measured the angle swept by the device tip from the starting position to the point of equilibrium. In order to test the presented analytical model and understand scaling phenomena, a family of devices with parametrically varied geometries was fabricated and assembled. The varied parameters rib length, rib spacing, spine thickness and device depth enabled the investigation of the effect of scaling on the magnitude of tip deflection, angular rotation and the exact geometry of deformation [32]. Typical as-drawn geometric parameters of the fabricated devices include 300, 400 and $500 \mu \mathrm{m}$ rib lengths, $75 \mu \mathrm{m}$ rib spacing, 10, 20 and $30 \mu \mathrm{m}$ spine thicknesses, and $80 \mu \mathrm{m}$ device depth. Using scanning electron microscopy (SEM) of rib cross-sections, the dimensions of actual fabricated device spine and rib thicknesses were found to be larger than design due to aspect ratio limitations of the photo-patternable silicone. The additional thickness was incorporated into the analytical model for a direct comparison of the theoretical predictions with the experimental data. Multiple sets of identical devices were tested to ensure repeatability.
Figure 6 shows a time-lapsed typical device movement during actuation. Once wetted, devices quickly moved to their equilibrium position (figures $6(b)$ and $(c)$ ). As water evaporated, the devices began to relieve the strain in the spine (figure $6(d)$ ). Devices returned to their undeformed state once dry (figure 6(e)).

\subsection{Device performance as a function of spine thickness and rib length}

Devices with the smallest feature size of $10 \mu \mathrm{m}$ spine thicknesses experienced resultant tip deflections in excess of $4 \mathrm{~mm}$ while unfurling $300^{\circ}$ (figures $7(a)$ and $(b)$ ). Devices exhibited forces per unit length ranging from $5.75 \mathrm{mN} \mathrm{m}^{-1}$ for $10 \mu \mathrm{m}$ spine thicknesses to $67.67 \mathrm{mN} \mathrm{m}^{-1}$ for $30 \mu \mathrm{m}$ spine thicknesses. Decreasing the spine thickness predictably increased the deflection of the device, but also increased the spread of the measured data. The data plots in figure 7(a) show an increase in the standard deviation as the spine thickness is decreased. This is partly due to test environment limitations. Since the test environment of an optical microscope for image capture cannot be guaranteed to provide evenly distributed light, devices with narrower spine geometries expressed the variation much more noticeably than wider devices, which did not deflect as much. The variation among a given spine thickness is rather constant relative to the total angular rotation and displacement. Devices with varied rib lengths also exhibit this variability due to the test environment. Also, as the rib 


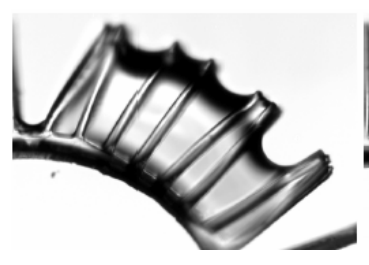

(a)

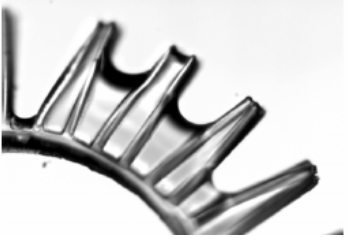

(b)

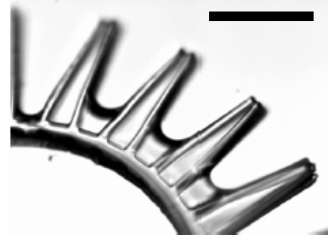

(c)

Figure 8. (a) Drying of liquid phase for the device whose ribs are not forced into contact. (b) At a certain time point devices undergo a breaking of symmetry due to uneven test environments which cause the water within some cells to evaporate faster than in others (i.e. non-uniform light intensity). Images are $2 \mathrm{~s}$ apart. The scale bar indicates $300 \mu \mathrm{m}$.

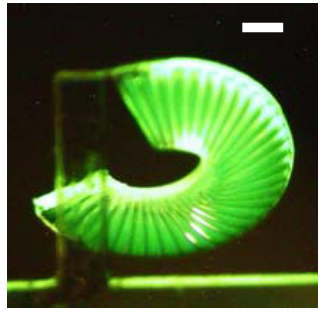

(a)

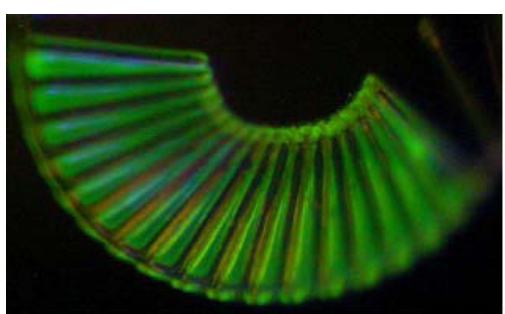

(b)

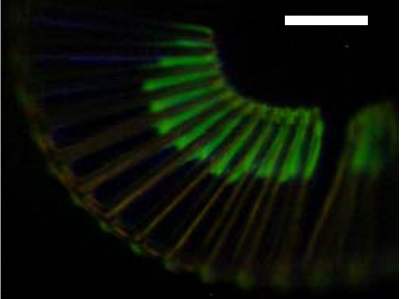

(c)

Figure 9. (a) Photograph of a device whose deflection is so extreme that the ribs are forced into contact. (b) Close-up of the device drying to show the liquid phase. The liquid is observed to dry toward the tip of the ribs, unlike drying toward the spine that is in figure 8 . (c) Due to the direction of the receding meniscus during drying, the device remained completely deformed at its maximum possible actuated position until all the liquid evaporated. The scale bar indicates $250 \mu \mathrm{m}$.

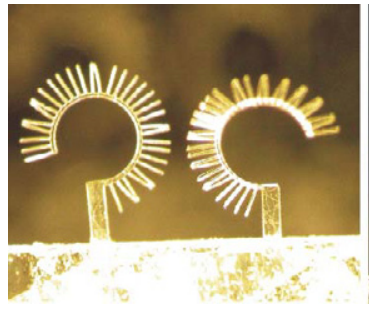

(a)

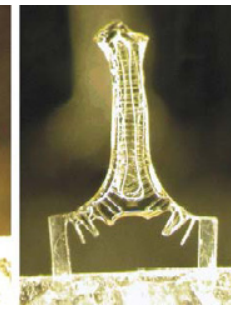

(b)

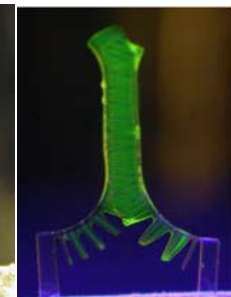

(c)

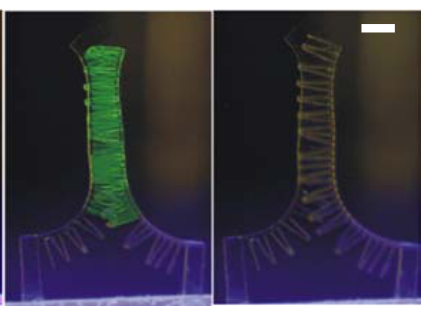

$(d)$

(e)

Figure 10. Two microactuators arranged so that the transient actuation due to evaporation leaves them in a different, stable configuration after they dry. (a) Two dry devices before adding water; $(b)$ after adding water independently to each structure, the devices interlock into a new, stable configuration. $(c)-(e)$. Fluorescein dye was used to show how the liquid phase dries, leaving a stable configuration. The scale bar indicates $400 \mu \mathrm{m}$.

length was increased, the ribs touched and prevented further deformation (figure $7(c)$ ). As seen in figure 7, the results of the analytical model fit the empirical data at silicone modulus values of 50-70 MPa. This matches previous observations that Young's modulus of PDMS can vary substantially depending on degrees of cross-linking, humidity and surface treatments (i.e. $\mathrm{O}_{2}$ plasma) $[33,34]$.

\subsection{Drying of liquid phase meniscus varies as a function of spine thickness and rib length}

In addition to the quantifiable differences in device performance as a function of rib length and spine thickness shown in figure 7 , additional visual observations were made as a result of the varied geometric parameters. This information is not captured by the analytical model since the model seeks to find the point of equilibrium and not the transient response.

The drying of the liquid phase was found to exhibit a varied response to rib length. For shorter rib lengths of $300 \mu \mathrm{m}$, the meniscus was observed to dry from the rib tips toward the spine (figure $8(a)$ ). At a certain time point, many devices underwent a breaking of symmetry due to varied light intensity in the test environment (figure $8(b)$ ). However, it is interesting to note that devices in which the meniscus dried toward the spine slowly returned to their starting position at a rate proportional to the evaporation of water.

For devices with longer rib lengths of $500 \mu \mathrm{m}$, the actuation is so extreme that the ribs are forced into contact (figures $9(a)$ and $(b)$ ). Note that, unlike the typical MEMS stiction of two cantilevers [35], the liquid is observed to dry toward the point contact at the tip of the ribs and away from the spine (figure $9(c)$ ). Since the liquid dries toward the tips of the ribs, the device remained completely deformed at its point of equilibrium until all the liquid evaporated. At that point the ribs immediately separated and the microactuator snapped back to its starting position.

Both of the drying phenomena described above can be seen in different parts of the device in figure 6 which had rib lengths of $400 \mu \mathrm{m}$. Variations in light intensity in the test environment are the most probable cause of uneven drying 
between the cells of the same device as it returned to its starting position.

By varying the geometry of the devices via the rib length, the meniscus can be programmed to dry toward the spine (for shorter rib lengths) or to dry toward the tip of the ribs (for longer rib lengths that touch at the tip during actuation). This also allows control of the speed with which a device returns to its original position; devices with shorter rib lengths return to their original position more slowly than those with longer rib lengths, which snap when the last drop of liquid holding two rib tips together dries.

\subsection{Bottom-up assembly: generating stable shapes with multiple actuators}

All of the devices discussed so far returned to their original position after evaporation was complete. However, this transient mechanism of transpiration actuation can be used to generate stable self-assembled configurations. Figure 10 shows two simple ribbed actuators fabricated near each other. Independently, each of these devices functioned as described above, transiently deflecting and then returning to their initial configuration upon drying. When the devices were positioned properly, there was a point at which the total water surface energy was reduced by forming a continuous water surface across the ends of both devices (figure 10(b)). As this occurred, the devices came into contact at certain points and interlocked (figures 10(b)-(e)). Once dry, the composite device stayed in its new configuration (figure 10(e)). This is due to the large areas which when come into contact during actuation (i.e. ribs along their entire length), experience stiction and thus cause the two actuators to stay together when dry. This shows that one can geometrically define devices such that during actuation, large surface areas come into contact and adhere due to stiction. For a single actuator, the only possible areas for stiction are the very small surface areas at the rib tips. That small area is not large enough to cause stiction for an individual device. Therefore, single actuating devices are able to return to their original positions without experiencing stiction.

Furthermore, additional devices were fabricated to show that a small $(<10)$ number of ribs could move large slabs of polymerized silicone. A curved slab of silicone $(0.5 \times$ $0.25 \mathrm{~mm}^{2}$ ) was moved approximately $2.5 \mathrm{~mm}$ by the evaporation of water between five ribs.

\section{Conclusion}

This paper presents transpiration actuation devices which utilize the pulling force of the surface tension of water on patterned ribs to deform structures in predictable ways. This type of actuation method is new and has been little studied as a micro or nanomechanical system. The structures presented in this paper exhibit large deformations due to surface tension. This method of actuation is being studied as a method of bottom-up self-assembly in which adjacent devices actuate and interlock into more complex and stable configurations. Moreover, the analytical model indicates that deflection relative to the size of the device was size invariant and that nanoscale structures would exhibit relative deformations and angular rotations as large as the microscale devices if the material's Young's modulus is scaled inversely with the device's spine thickness. The deflection profiles of the actuators might be further tuned with environmental controls such as light or heat sources and with more interesting geometries achieved by staggering or varying spine geometry or rib length throughout a single device. These variables could be used to change the speed, location and direction of actuation. The presented actuation scheme provides a possible route toward embedding evaporation-induced actuation into common materials. This mechanism is being explored to create two-dimensional programmable sheets of material that deform as a function of humidity.

\section{Acknowledgments}

The authors would like to thank the Michigan Nanofabrication Facility and the NSF ERC for Wireless Integrated MicroSystems (WIMS) staff. We would like to thank Professor Noel C Perkins, Professor Yogesh Gianchandani, Dr Sachin Goyal and Meng-Ping Chang for their valuable feedback. We would also like to thank Tzeno Galchev for various discussions. This work was funded by the National Science Foundation.

\section{References}

[1] Aksay I A, Groves J T, Gruner S M, Lee P C, Prud'homme R K, Shih W-H, Torquato S and Whitesides G M 1996 Smart materials systems through mesoscale patterning Proc. SPIE 2716 280-91

[2] Warneke B, Last M, Liebowitz B and Pister K S J 2001 Smart Dust: communicating with a cubic-millimeter computer Computer 3444

[3] Roundy S, Leland E S, Baker J, Carleton E, Reilly E, Lai E, Otis B, Rabaey J M, Wright P K and Sundararajan V 2005 Improving power output for vibration-based energy scavengers IEEE Pervasive Computing 428

[4] Roundy S 2005 On the effectiveness of vibration-based energy harvesting J. Intell. Mater. Syst. Struct. 16809

[5] Qidwai M A, Thomas J P, Kellogg J C and Baucom J 2004 Energy harvesting concepts for small electric unmanned systems Proc. SPIE 5387 84-95

[6] Gracias D H, Kavthekar V, Love J C, Paul K E and Whitesides G M 2002 Fabrication of micrometer-scale, patterned polyhedra by self-assembly Adv. Mater. 14235

[7] Green P W, Syms R R A and Yeatman E M 1995 Demonstration of three-dimensional microstructure self-assembly J. Microelectromech. Syst. 4170

[8] Syms R R A, Yeatman E M, Bright V M and Whitesides G M 2003 Surface tension-powered self-assembly of microstructures - the state-of-the-art J. Microelectromech. Syst. 12387

[9] Bohringer K F, Srinivasan U and Howe R T 2001 Modeling of capillary forces and binding sites for fluidic self-assembly 14th IEEE Int. Conf. on Micro Electro Mechanical Systems (MEMS '01) (Interlaken, Switzerland) pp 369-374

[10] Morris C J, Stauth S A and Parviz B A 2005 Self-assembly for microscale and nanoscale packaging: steps toward self-packaging IEEE Trans. Compon. Packag. Manuf. Technol. B 28600

[11] Srinivasan U, Liepmann D and Howe R T 2001 Microstructure to substrate self-assembly using capillary forces J. Microelectromech. Syst. 1017

[12] Xiaorong X, Sheng-Hsiung L and Bohringer K F 2004 Geometric binding site design for surface-tension driven self-assembly 17th IEEE Int. Conf. on Micro Electro 
Mechanical Systems (MEMS '04) (New Orleans, LA, USA) p 9-12

[13] Kim C-J 2003 The use of surface tension for the design of MEMS actuators Nanotribology: Critical Assessment and Research Needs ed S M Hsu and Z C Ying (Boston: Kluwer)

[14] Regan B C, Aloni S, Jensen K and Zettl A 2005 Surface-tension-driven nanoelectromechanical relaxation oscillator Appl. Phys. Lett. 86123119

[15] Boncheva M and Whitesides G M 2005 Templated self-assembly: formation of folded structures by relaxation of pre-stressed, planar tapes Adv. Mater. 17553

[16] Gracias D H, Tien J, Breen T L, Hsu C and Whitesides G M 2000 Forming electrical networks in three dimensions by self-assembly Science $\mathbf{2 8 9} 1170$

[17] Stoykovich M P, Cao H B, Yoshimoto K, Ocola L E and Nealey P F 2003 Deformation of nanoscopic polymer structures in response to well-defined capillary forces Adv. Mater. 151180

[18] Slatyer R O 1967 Plant-Water Relationships (New York: Academic) p 212

[19] Sutcliffe J 1968 Plants and Water vol 14 The Institute of Biology's Studies in Biology (London: Edward Arnold) pp 72-76

[20] Trevena D H 1987 Cavitation and Tension in Liquids (Philadelphia: Adam Hilger) pp 110-111

[21] Tyree M T and Zimmerman M H 2002 Xylem Structure and the Ascent of Sap (New York: Springer) pp 49-64

[22] Borno R T and Maharbiz M M 2005 A distributed actuation method based on Young-Laplace forces Dig. of Tech. Papers: The 13th Int. Conf. on Solid-State Sensors, Actuators and Microsystems (Transducers'05) (Seoul, South Korea) pp 125-128

[23] Ursprung A 1915 Über die Kohäsion des Wasser in Farnannulus Berichte der Deutsch Botanischen Gesellschaft 33 152-62
[24] Baroud C N and Willaime H 2004 Multiphase flows in microfluidics C. R. Phys. 5 547-55

[25] Stone H A and Kim S 2001 Microfluidics: basic issues, applications, and challenges AIChE J. 47 1250-4

[26] Chanliaud E, Burrows K, Jeronimidis G and Gidley M 2002 Mechanical properties of primary plant cell wall analogues Planta 215989

[27] Senturia S D 2000 Microsystem Design (Dordrecht: Kluwer) pp 240-4

[28] Weaver J W, Timoshenko S P and Young D H 1990 Vibration Problems in Engineering 5th edn (New York: Wiley) pp 518-20

[29] Bower D L 2002 An Introduction to Polymer Physics (Cambridge: Cambridge University Press) pp 164-76

[30] Maneschy C E, Massoudi M and Velloso V R 1993 Dynamic elastic solutions in neo-Hookean and Mooney-Rivlin materials Int. J. Nonlinear Mech. 28531

[31] Gere J M 1996 Bending stresses in beams The Engineering Handbook ed R C Dorf (Boca Raton, FL: CRC Press) pp 52-59

[32] Goyal S, Perkins N C and Lee C L 2005 Nonlinear dynamics and loop formation in Kirchoff rods with implications to the mechanics of DNA and cables J. Comput. Phys. $209371-389$

[33] Armani D, Liu C and Aluru N 1999 Re-configurable fluid circuits by PDMS elastomer micromachining 12th IEEE Int. Conf. on Micro Electro Mechanical Systems (MEMS '99) (Orlando, FL, USA) pp 222-227

[34] Carillo F, Gupta S, Balooch M, Marshall S J, Marshall G W, Pruitt L and Puttlitz C M 2005 Nanoindentation of polydimethylsiloxane elastomers: effect of crosslinking, work of adhesion, and fluid environment on elastic modulus J. Mater. Res. 202820

[35] Maboudian R and Howe R T 1997 Critical review: adhesion in surface micromechanical structures J. Vac. Sci. Technol. B 151 